\title{
Optimum method for administration of biosynthetic human growth hormone: a randomised crossover trial of an Auto Injector and a pen injection system
}

\author{
R Stanhope, A Albanese, L Moyle, G Hamill
}

\begin{abstract}
The use of optimum conventional growth hormone administration, using a growth hormone vial combined with an Auto Injector, was compared with a pen injection system using a cartridge of growth hormone. In both methods of administration the concentration of growth hormone was $16 \mathrm{IU} / \mathrm{ml}$. Thirty patients ( 22 boys, eight girls) who had all previously been treated with growth hormone (4 IU/ml) administered using needles and syringes (without an Auto Injector) were randomised into receiving one of either treatment for three months and then crossed over for a further three months. Fourteen patients (10 boys, four girls) initially received KabiVial 16 IU $/ \mathrm{ml}$ combined with an Auto Injector while 16 patients (12 boys, four girls) were treated with KabiPen $16 \mathrm{IU} / \mathrm{ml}$. Mean age in both groups was $\mathbf{9 . 6}$ years. The majority of patients in both groups were treated with a regimen of either 15 or 20 units $/ \mathrm{m}^{2} /$ week as a daily subcutaneous injection. Of the 30 patients who started in this trial, two who commenced using an Auto Injector refused to change to a pen system and were excluded from further analysis. When scored on a scale of -5 to +5 general convenience when changing from an Auto Injector to the KabiPen decreased from $+4 \cdot 7$ to $+1 \cdot 0$. When assessed for pain, the Auto Injector group scored $+4 \cdot 7$, which decreased to $-\mathbf{0 . 2}$ (more painful) for the pen. At the end of the trial 23 patients $(82 \%)$ chose to continue with the KabiVial/Auto Injector combination as they found this less painful and the child did not see the needle or need to insert the needle manually. Five patients $(18 \%)$ continued with the KabiPen as they considered the device smaller and easier to use. The accuracy of dosing using KabiVial was $100 \%$ compared with the range of $88 \%$ to $111 \%$ using KabiPen as the latter was available only in 0.5 unit increments. No growth hormone was wasted using KabiVial, although a mean of 0.6 units was wasted with every 16 IU cartridge in the KabiPen system.

It is concluded that patients should be able to contribute to the choice of growth hormone delivery systems and that newer methods need careful assessment.
\end{abstract}

\section{(Arch Dis Child 1992;67:994-997)}

30 Guilford Street,

CIN

R Stanhope

A Albanese

L Moyle

G Hamill

Correspondence to:

Dr Stanhope.

Accepted 27 March 1992
Biosynthetic human growth hormone has traditionally been administered using a powdered growth hormone preparation in a vial to which diluent is added before the resulting solution is drawn up into a smaller syringe before injection. This is a relatively inconvenient form of preparation. Kabi Pharmacia have developed a new range of convenience products which are superior in that the growth hormone powder and water are present in the same applicator and are mixed by closing a syringe barrel (fig 1). Administration is by either needles and syringes or as a cartridge in a pen injection system. ${ }^{1}$ Both of these new formulations of biosynthetic human growth hormone (Genotropin) are prepared in an identical concentration of $16 \mathrm{IU} / \mathrm{ml}$.

Previous trials of a pen injection system against conventional application by needles and syringes have been biased because the volume of injection has been unequal when comparing the two systems, ${ }^{1-3}$ which may be of particular significance when assessing pain. Although a pen injection system may offer a significant improvement in convenience, the patient still has manually to insert the needle subcutaneously. However, pen injection systems have not been evaluated against optimum treatment using an Auto Injector (which uses a $1 \mathrm{ml}$ syringe and needle), a spring loaded device which automatically inserts the needle subcutaneously and delivers the injection.

The dose of growth hormone administered should be related to the patient's size. ${ }^{4}$ Concern has previously been expressed about the dose regimen using a KabiPen injection system as only 0.5 unit incrèments are available up to a maximum of only 4.0 units, which severely limits its use in very small children and adolescents respectively, as well as reducing the dosage accuracy. ${ }^{5}$

We have used KabiVial (16 IU/ml) in combination with an Auto Injector and compared this device to a KabiPen (16 IU $/ \mathrm{ml}$ ) in order to determine which method was preferred by the patients and their parents. In addition we have examined the accuracy of dose administration and the potential wastage of growth hormone between the two systems.

Patients and methods

Thirty patients were randomly selected from the Growth Clinic at the Hospital for Sick Children, Great Ormond Street, all of whom were being treated with biosynthetic human growth hormone (Genotropin 12 IU, Kabi Pharmacia) which is a vial of growth hormone reconstituted in a solution of $4 \mathrm{IU} / \mathrm{ml}$. The majority of patients had isolated idiopathic growth hormone deficiency $(n=18)$; the other diagnostic groups were idiopathic multiple 


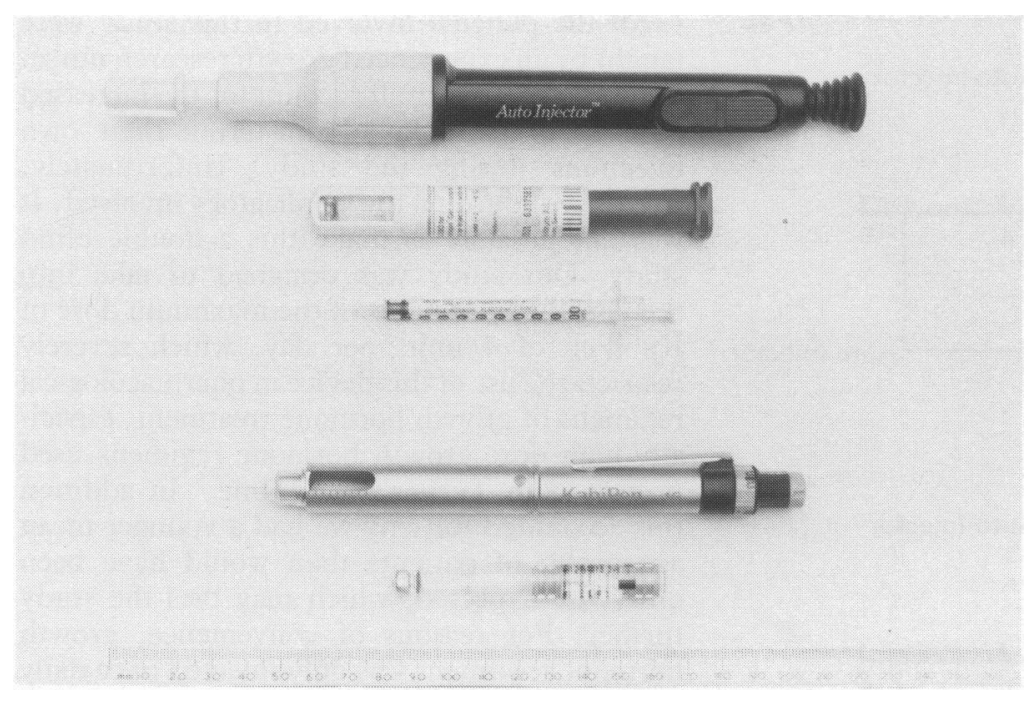

Figure 1 At the top of the figure is an Auto Injector and $1 \mathrm{ml}$ syringe and needle with a KabiVial $16 \mathrm{IU} / \mathrm{ml}$. At the bottom of the figure is KabiPen with a KabiPen $16 \mathrm{IU} / \mathrm{ml}$ cartridge. A scale in $\mathrm{cm}$ is indicated. pituitary hormone deficiency $(n=3)$, hypopituitarism after cranial irradiation $(n=4)$, and dysmorphic syndromes $(n=5)$. One of the latter children had Ehler-Danlos syndrome with easy bruising as part of his symptoms. Growth hormone in all the patients was administered by needles and syringes and none had previously used a pen injection system or an Auto Injector. All patients were selected by receiving a dose regimen of under $4 \mathrm{IU}$ per day (the maximum dose which can be administered by KabiPen). After obtaining parental consent to participate in this study, patients were randomised to either three months of growth hormone administered by a pen injection system (KabiPen) or using KabiVial in combination with an Auto Injector (Owen Mumford Ltd). Mean age in the two groups was 9.7 years (range $3.3-13.7$ ) in one group and 9.6 years (range $5 \cdot 1-16.6$ ) in the other. Sex distribution was similar in the two groups: 14 patients (10 boys, four girls) and 16 patients ( 12 boys, four girls) respectively. Surface area was similar in both groups: $1.0 \mathrm{~m}^{2}$ (range $0.5-1.8$ ) and $0.95 \mathrm{~m}^{2}$ (range $0.6-1.5$ ).

After a three month period all patients completed a questionnaire and then changed to a further three months of treatment with the alternative method of administration and completed a further questionnaire. These were completed in the presence of a growth research nurse (GH) and both pain of injection and general convenience were rated on a scale of -5 to +5 . At the end of the six month period the children were allowed to continue with whichever treatment method they preferred.

Growth hormone administration was calculated as a weekly total dose related to surface area and divided equally into seven daily subcutaneous injections. The dose regimen of growth hormone administration was similar in both groups: the majority received either $15 \mathrm{IU} /$ $\mathrm{m}^{2} /$ week ( $\mathrm{n}=4$ in both groups) or $20 \mathrm{IU} / \mathrm{m}^{2} /$ week ( $n=7$ in both groups). A few patients received higher dose regimens of 25 or $30 \mathrm{IU} /$ $\mathrm{m}^{2} /$ week but were similarly distributed in both treatment groups. The daily dose of growth hormone was calculated and allocated to the closest 0.5 unit increment available when using the pen system, and the difference in dose allocation between KabiVial and KabiPen was calculated as a percentage. Growth hormone remaining in the cartridge of the KabiPen, after the last full daily dose was administered, was calculated. As these products are designed for patient convenience, only one injection was permitted for each day.

Ethical committee approval was obtained for this study. Statistical analysis was by paired $t$ test.

\section{Results}

Thirty patients started in this study, but after the first three months two patients refused to change from KabiVial/Auto Injector. Both children found the main advantage of an Auto Injector was that they did not have to insert the needle manually. These two patients were excluded from the rest of the analysis. At the end of the study 23 patients $(82 \%)$ wished to continue with KabiVial/Auto Injector. The predominant reasons given were that this was less painful $(n=16)$, the needle was not seen $(n=4)$, and the needle was not inserted manually $(n=3)$. Five patients $(18 \%)$ elected to continue with the KabiPen. This was considered easier to use, especially as it was smaller $(n=3)$, more 'gentle' ( $n=1$, this was the child with EhlerDanlos syndrome who had easy bruising), and less painful $(n=1)$. This result was similar irrespective of which group the patients were initially randomised to. Of those continuing with KabiVial/Auto Injector after the trial had finished, nine patients (75\%) were from the group who initially received an Auto Injector, whereas 13 patients $(81 \%)$ initially received the KabiPen.

Ten children (36\%) initially gave their own injections before this trial commenced, and these were equally distributed between the two groups. After the six months of this trial, 16 children $(57 \%)$ gave their own injections; 12 out of 23 $(52 \%)$ of those continuing with a KabiVial/Auto Injector and four out of five $(80 \%)$ of those continuing on the KabiPen injector system. The results of the patients' scoring of the different methods of growth hormone application are given in fig 2 . When scored for general convenience, changing from the Auto Injector to the KabiPen, the score decreased from $+4 \cdot 7$ to $+1.0(p<0.001)$ and when scored for pain the decrease was from $+4 \cdot 0$ to $-0.2(p<0.001)$.

The accuracy of dosage administration was $100 \%$ using KabiVial whereas the range of dose variation was between $88 \%$ and $111 \%$ using KabiPen, which relates to the fixed 0.5 unit increments between dose schedules. No wastage of growth hormone occurred using KabiVial as at the end of the vial the remaining growth hormone was topped up using a new vial. However using the pen cartridge system the mean quantity of growth hormone remaining at the end of the cartridge was 0.6 units (range $0-1 \cdot 0)$. 

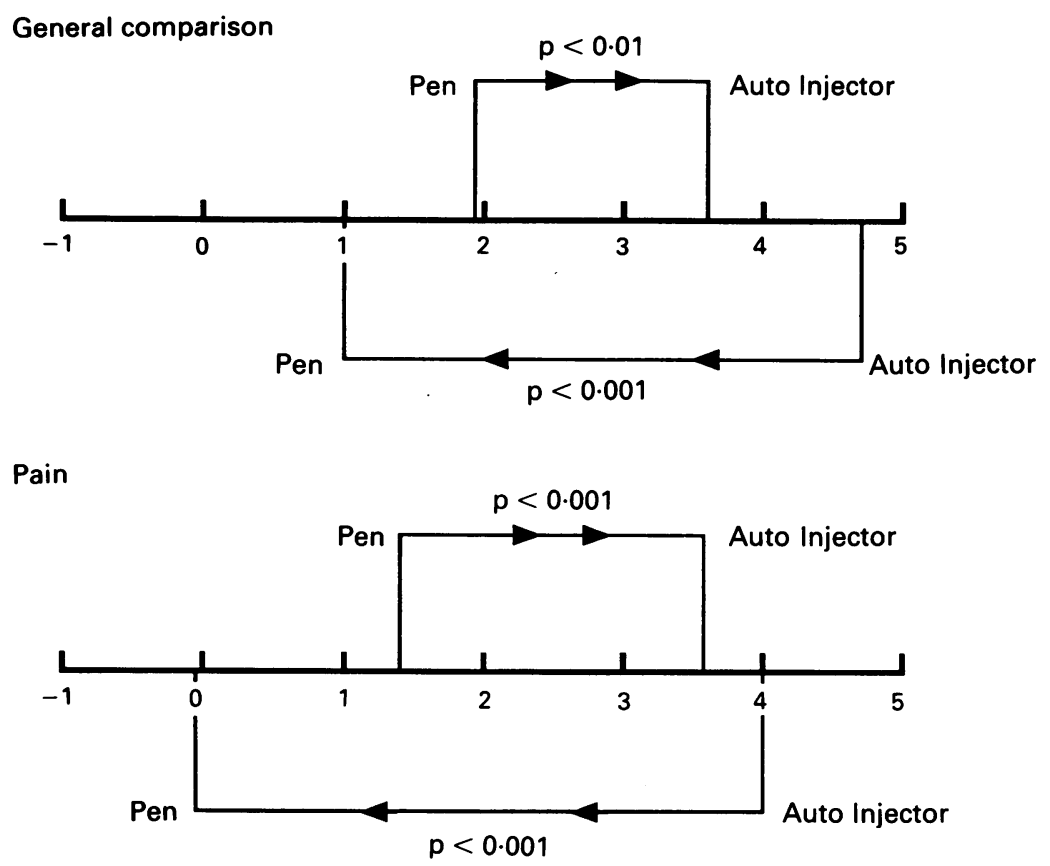

Figure 2 Graded patient scores $(-5$ to +5$)$ after each three month period, with the baseline comparison made to the original method of growth hormone injection using needles and syringes.
All the patients involved in this study were taught by an experienced growth research nurse. Certainly this may well account for the increased number of children administering their own injections during the study. Unfortunately, from the nature of the applicators involved, it was not possible to make this a double blind study. Our study was designed to take into account the limitations of the maximum dose of KabiPen of 4 units per day, which severely restricts the use of this device in pharmacological regimens of growth hormone treatment, especially high dose growth hormone regimens used in girls with Turner's syndrome. ${ }^{5}$ In addition this accounted for why we had a younger mean age group of patients than would have been otherwise expected, which may bias the study further. For reasons of convenience, growth hormone treatment was only injected once daily and this led to a residue of growth hormone in the KabiPen cartridge whereas there was no corresponding waste using a vial. Of course, there is the possibility of using different dosages of growth hormone on different days of the week in order to avoid wastage but this would imply a complicated regimen that may be counterproductive in terms of compliance.

Certainly the most important decision of which applicator to use, in terms of convenience, should be made by the child/parent. Although growth hormone from a vial combined with an Auto Injector may be more complicated to prepare, the simplicity and passivity of the injection technique outweighs the advantages of a pen injector system. Additionally, in terms of dosage consideration, a vial is more accurate and less wasteful of expensive resources. Of course the KabiPen injection system would be improved if doses greater than 4 units could be administered and if dose increments were acceptable in less than 0.5 units. It is unlikely that more than one growth hormone injection per day ${ }^{6}$ is necessary to optimise growth response and so the convenience of a relatively portable pen device becomes minimal, in contrast to the experience of an insulin pen injection system in the treatment of diabetes mellitus. Great care should be taken in advising patients about injection techniques and we recommend that children/parents are shown the alternative methods available and then they are able to make their own choice. This may well be of great significance for compliance to growth hormone treatment, which is probably far worse than had previously been appreciated (S L Smith, C G D Brook. Paper presented to the British Society for Paediatric Endocrinology, November 1991). The KabiPen injector combined with an Auto Injector function would probably be the optimum device for delivering growth hormone. However, whichever method is used, we believe that the involvement of a nurse specialist, time taken to explain treatment options, teaching and follow up, remain an essential element of the therapeutic regimen.

G Hamill was funded by the Child Growth Foundation. L Moyle was supported by Kabi Pharmacia (UK). W'e are grateful to Serono (UK) for financial assistance in the preparation of the manuscript. more convenient using both KabiVial and KabiPen than older growth hormone multidose vials. 
1 Jorgensen J O, Mooller J, Jensen F S, Joorgensen J T, Christiansen J S. Growth hormone administration by means of an injection pen. Pharmacol Toxicol 1989;65:96-9. 2 Albertsson-Wikland K. Simplified growth hormone therapy-first clinical experience with the KabiPen. Acta therapy-first clinical experience with
Paediatr Scand $1988 ; 343$ (suppl): $103-6$.

3 Gluckman PD, Cutfield WS. Evaluation of a pen injector system for growth hormone treatment. Arch Dis Child 1991;66:686-8.

4 Smith PJ, Hindmarsh PC, Brook CGD. Contribution of dose and trequency of administration to the therapeutic effect of growth hormone. Arch Dis Child 1988;63:491-4.

5 Hamill G, Stanhope R. Evaluation of a pen injector system for growth hormone treatment. Arch Dis Child 1991;66:

6 Hindmarsh PC, Stanhope R, Preece MA, Brook CGD. Frequency of administration of growth hormone-an important factor in determining growth response to exogenous growth hormone. Hormone Res 1990;33 (supp 4):83-9.

Cutting the corpus callosum for intractable epilepsy

There can be few clinical problems more distressing for families and doctors then truly intractable epilepsy. The medical approach to the problem has been admirably summarised by Aicardi. ${ }^{1}$ What about surgery? ${ }^{2}$ Few of these patients are suitable for conventional resective surgery but there is increasing interest in the use of anterior callostomy. A recent report from Montreal (Hirokazu Oguni and colleagues, Annals of Neurology 1991;30:357-64) describes the results of this operation in 43 patients followed up for at least a year. The youngest was 7 years old and the oldest 60 but, unfortunately, detailed information about the ages of the patients is not given. Nevertheless, from the types of epilepsy being treated it is reasonable to assume that many were children. (It would be very helpful if research workers presenting data on a mixed population of children and adults would give specific information about the children. A recent report on the withdrawal of antiepileptic treatment ${ }^{3}$ was almost impossible to analyse from the paediatric point of view because of the lack of such information.)

There was no operative mortality in this series but postoperative complications of varying severity occurred in 16 of the 43 patients. None gave rise to permanent morbidity and no clinically significant longlasting neurological disturbance resulted from the surgery. The results were better with more extensive surgery and it is now the policy in Montreal to divide the anterior two thirds of the corpus callosum.

The results are encouraging, the effect on seizure control being 'good' in 33\% and 'worthwhile' in 56\%. It might be expected that callostomy would benefit most those with seizures of focal origin with secondary generalisation and there was some evidence in this series that this was so but significant benefit was also seen in apparently primarily generalised epilepsy. Only six of the 43 patients were of normal intelligence and many were severely retarded. Improvement after operation occurred at all levels of intelligence. Small increases in measured IQ were found in those whose epilepsy improved. Of particular interest to paediatricians is the finding that eight of 14 patients with the Lennox-Gastaut syndrome gained significant benefit from the operation.

The apparent success of this operation poses considerable logistic problems. Neurosurgeons with a specific interest in epilepsy surgery are few, as are centres with the appropriate staffing and facilities for assessment of these patients but patients who might be considered for surgery are many. The implications for the organisation of services for children with epilepsy are potentially very great.

1 Aicardi J. Clinical approach to the management of intractable epilepsy. Dev Med Child Neurol 1988;30:429-40.

2 Polkey CE. Surgery for epilepsy. Arch Dis Child 1989;64:185-7. study of antiepileptic drug withdrawal in patients in remission. Lancet 1991;337: study of 